\title{
FERMI-LAT DISCOVERY OF GeV GAMMA-RAY EMISSION FROM THE YOUNG SUPERNOVA REMNANT CASSIOPEIA A
}

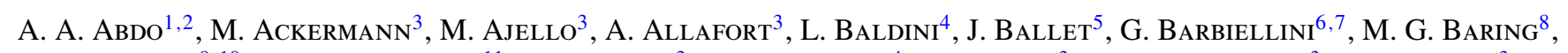
D. Bastieri ${ }^{9,10}$, B. M. Baughman ${ }^{11}$, K. Bechtol ${ }^{3}$, R. Bellazzini ${ }^{4}$, B. Berenji ${ }^{3}$, R. D. Blandford ${ }^{3}$, E. D. Bloom ${ }^{3}$, E. Bonamente ${ }^{12,13}$, A. W. Borgland ${ }^{3}$, J. Bregeon ${ }^{4}$, A. Brez ${ }^{4}$, M. Brigida ${ }^{14,15}$, P. Bruel ${ }^{16}$, R. Buehler ${ }^{3}$, T. H. Burnett ${ }^{17}$, G. Busetto ${ }^{9,10}$, G. A. Caliandro ${ }^{18}$, R. A. Cameron ${ }^{3}$, P. A. Caraveo ${ }^{19}$, J. M. Casandian ${ }^{5}$, C. CeCchi ${ }^{12,13}$, Ö. Çelik ${ }^{20,21,22}$, E. Charles $^{3}$, S. Chaty ${ }^{5}$, A. Chekhtman ${ }^{1,23}$, C. C. Cheung ${ }^{1,2}$, J. Chiang ${ }^{3}$, A. N. Cillis ${ }^{20}$, S. Ciprini ${ }^{13}$, R. Cllaus ${ }^{3}$, J. Cohen-Tanugi ${ }^{24}$, J. Conrad ${ }^{25,26,58}$, S. Corbel ${ }^{5}$, F. De Palma ${ }^{14,15}$, S. W. Digel ${ }^{3}$, M. Dormody ${ }^{27}$, E. do Couto E Silva ${ }^{3}$, P. S. Drell ${ }^{3}$, R. Dubois ${ }^{3}$, D. Dumora ${ }^{28,29}$, Y. Edmonds ${ }^{3}$, C. FARnier ${ }^{24}$, C. FAVuzzi ${ }^{14,15}$, S. J. Fegan ${ }^{16}$, E. C. Ferrara ${ }^{20}$,

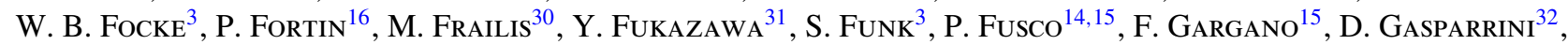
N. Gehrels ${ }^{20,33,34}$, S. Germani ${ }^{12,13}$, G. Giavitto ${ }^{6,7}$, N. Giglietto ${ }^{14,15}$, F. Giordano ${ }^{14,15}$, T. GlanZman ${ }^{3}$, G. Godfrey ${ }^{3}$, I. A. Grenier ${ }^{5}$, M.-H. Grondin ${ }^{28,29}$, J. E. Grove ${ }^{1}$, L. Guillemot ${ }^{35}$, S. Guiriec ${ }^{36}$, Y. Hanabata ${ }^{31}$, E. Hays ${ }^{20}$, A. K. HardinG ${ }^{20}$,

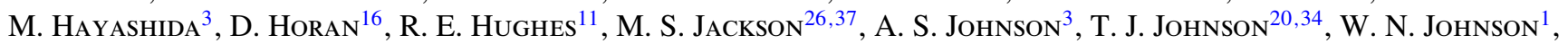

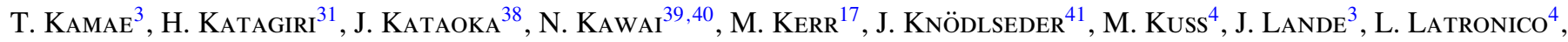

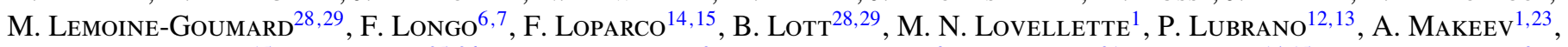
M. N. Mazziotta ${ }^{15}$, C. Meurer ${ }^{25,26}$, P. F. Michelson ${ }^{3}$, W. Mitthumsiri ${ }^{3}$, T. Mizuno ${ }^{31}$, C. Monte $^{14,15}$, M. E. Monzani ${ }^{3}$,

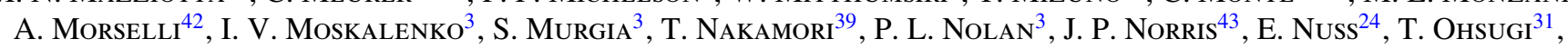
A. Okumura ${ }^{44}$, N. Omodei ${ }^{4}$, E. Orlando ${ }^{45}$, J. F. Ormes ${ }^{43}$, D. Paneque ${ }^{3}$, J. H. Panetta ${ }^{3}$, V. Pelassa ${ }^{24}$, M. Pepe ${ }^{12,13}$,

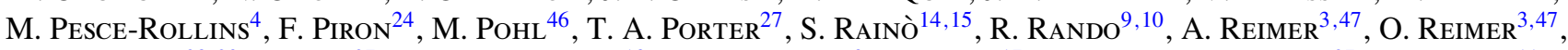
T. Reposeur ${ }^{28,29}$, S. Ritz ${ }^{27}$, A. Y. Rodriguez ${ }^{18}$, R. W. Romani ${ }^{3}$, M. Roth ${ }^{17}$, H. F.-W. SAdrozinSki ${ }^{27}$, A. SANDER ${ }^{11}$, P. M. Saz Parkinson ${ }^{27}$, J. D. Scargle ${ }^{48}$, C. Sgrò ${ }^{4}$, E. J. Siskind ${ }^{49}$, D. A. Smith ${ }^{28,29}$, P. D. SMith ${ }^{11}$, P. Spinelli ${ }^{14,15}$, M. S. Strickman ${ }^{1}$, D. J. Suson ${ }^{50}$, H. TAJima ${ }^{3}$, T. TAKAhashi ${ }^{51}$, T. TANAKA ${ }^{3}$, J. B. ThaYeR ${ }^{3}$, J. G. ThaYeR ${ }^{3}$, D. J. ThOMPSON ${ }^{20}$,

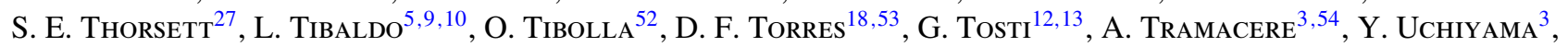

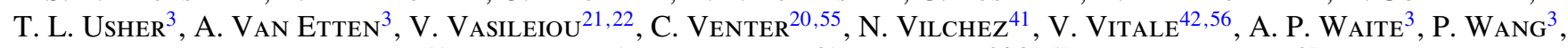
B. L. WineR ${ }^{11}$, K. S. WOOD ${ }^{1}$, R. YAMAZAKI ${ }^{31}$, T. YLINEN ${ }^{26,37,57}$, AND M. ZIEGLER ${ }^{27}$

${ }^{1}$ Space Science Division, Naval Research Laboratory, Washington, DC 20375, USA

${ }^{2}$ National Research Council Research Associate, National Academy of Sciences, Washington, DC 20001, USA

${ }^{3}$ W. W. Hansen Experimental Physics Laboratory, Kavli Institute for Particle Astrophysics and Cosmology, Department of Physics and SLAC National Accelerator Laboratory, Stanford University, Stanford, CA 94305, USA; funk@slac.stanford.edu, uchiyama@ slac.stanford.edu

${ }^{4}$ Istituto Nazionale di Fisica Nucleare, Sezione di Pisa, I-56127 Pisa, Italy

${ }^{5}$ Laboratoire AIM, CEA-IRFU/CNRS/Université Paris Diderot, Service d'Astrophysique, CEA Saclay, 91191 Gif sur Yvette, France

${ }^{6}$ Istituto Nazionale di Fisica Nucleare, Sezione di Trieste, I-34127 Trieste, Italy ${ }^{7}$ Dipartimento di Fisica, Università di Trieste, I-34127 Trieste, Italy

8 Department of Physics and Astronomy, Rice University, MS-108, P.O. Box 1892, Houston, TX 77251, USA

${ }_{9}^{9}$ Istituto Nazionale di Fisica Nucleare, Sezione di Padova, I-35131 Padova, Italy

${ }^{10}$ Dipartimento di Fisica "G. Galilei," Università di Padova, I-35131 Padova, Italy
${ }^{11}$ Department of Physics, Center for Cosmology and Astro-Particle Physics, The Ohio State University, Columbus, OH 43210, USA

${ }_{12}^{12}$ Istituto Nazionale di Fisica Nucleare, Sezione di Perugia, I-06123 Perugia, Italy

${ }^{13}$ Dipartimento di Fisica, Università degli Studi di Perugia, I-06123 Perugia, Italy

${ }^{14}$ Dipartimento di Fisica “M. Merlin” dell’Università e del Politecnico di Bari, I-70126 Bari, Italy

${ }^{15}$ Istituto Nazionale di Fisica Nucleare, Sezione di Bari, 70126 Bari, Italy

${ }^{16}$ Laboratoire Leprince-Ringuet, École polytechnique, CNRS/IN2P3, Palaiseau, France

${ }^{17}$ Department of Physics, University of Washington, Seattle, WA 98195-1560, USA

${ }^{18}$ Institut de Ciencies de l'Espai (IEEC-CSIC), Campus UAB, 08193 Barcelona, Spain

${ }^{19}$ INAF-Istituto di Astrofisica Spaziale e Fisica Cosmica, I-20133 Milano, Italy ${ }^{20}$ NASA Goddard Space Flight Center, Greenbelt, MD 20771, USA

${ }^{21}$ Center for Research and Exploration in Space Science and Technology (CRESST) and NASA Goddard Space Flight Center, Greenbelt, MD 20771, USA

${ }^{22}$ Department of Physics and Center for Space Sciences and Technology, University of Maryland Baltimore County, Baltimore, MD 21250, USA

${ }^{23}$ George Mason University, Fairfax, VA 22030, USA

${ }^{24}$ Laboratoire de Physique Théorique et Astroparticules, Université Montpellier 2, CNRS/IN2P3, Montpellier, France

${ }^{25}$ Department of Physics, Stockholm University, AlbaNova, SE-106 91 Stockholm, Sweden

26 The Oskar Klein Centre for Cosmoparticle Physics, AlbaNova, SE-106 91 Stockholm, Sweden

${ }^{27}$ Santa Cruz Institute for Particle Physics, Department of Physics and Department of Astronomy and Astrophysics, University of California at Santa Cruz, Santa Cruz, CA 95064, USA

${ }^{28}$ Université de Bordeaux, Centre d'Études Nucléaires Bordeaux Gradignan, UMR 5797, Gradignan 33175, France

${ }^{29}$ CNRS/IN2P3, Centre d'Études Nucléaires Bordeaux Gradignan, UMR 5797, Gradignan 33175, France

${ }^{30}$ Dipartimento di Fisica, Università di Udine and Istituto Nazionale di Fisica Nucleare, Sezione di Trieste, Gruppo Collegato di Udine, I-33100 Udine, Italy

${ }^{31}$ Department of Physical Sciences, Hiroshima University, Higashi-Hiroshima, Hiroshima 739-8526, Japan

32 Agenzia Spaziale Italiana (ASI) Science Data Center, I-00044 Frascati (Roma), Italy

33 Department of Astronomy and Astrophysics, Pennsylvania State University, University Park, PA 16802, USA

${ }^{34}$ Department of Physics and Department of Astronomy, University of Maryland, College Park, MD 20742, USA

${ }^{35}$ Max-Planck-Institut für Radioastronomie, Auf dem Hügel 69, 53121 Bonn, Germany

${ }^{36}$ Center for Space Plasma and Aeronomic Research (CSPAR), University of Alabama in Huntsville, Huntsville, AL 35899, USA

${ }^{37}$ Department of Physics, Royal Institute of Technology (KTH), AlbaNova, SE-106 91 Stockholm, Sweden

${ }^{38}$ Waseda University, 1-104 Totsukamachi, Shinjuku-ku, Tokyo 169-8050, Japan 


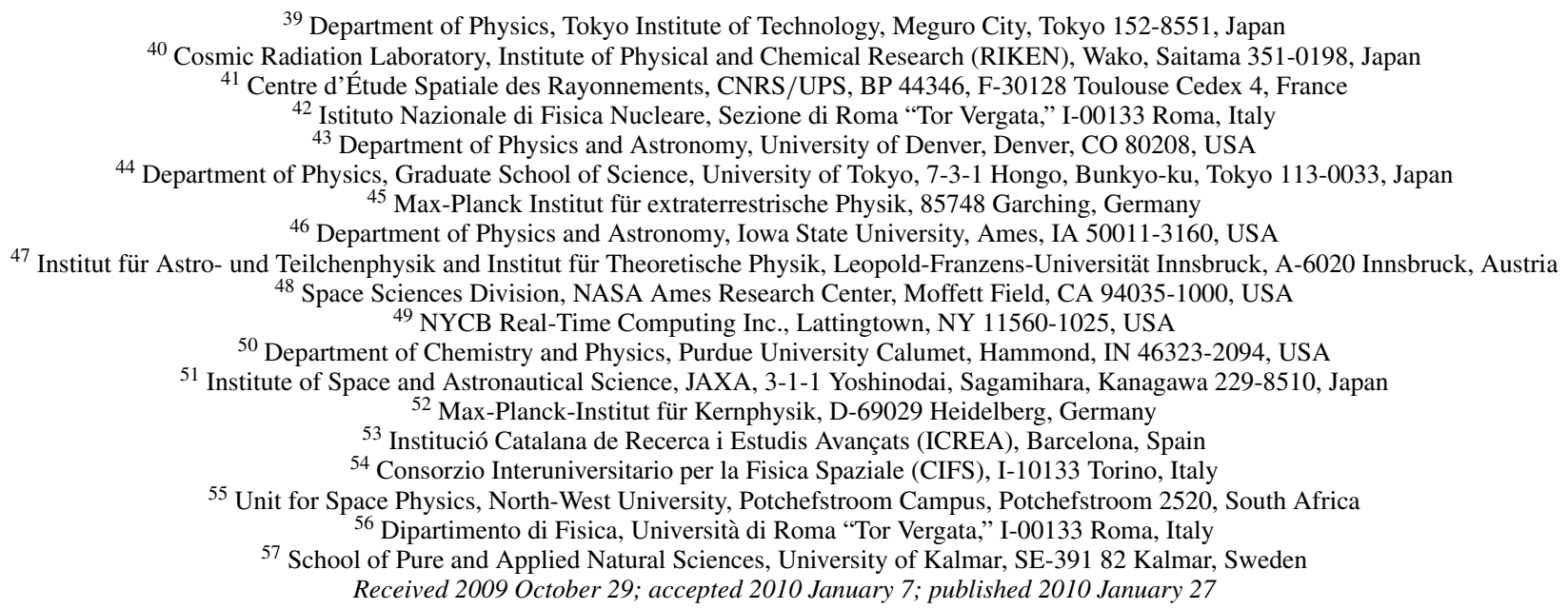

\begin{abstract}
We report on the first detection of GeV high-energy gamma-ray emission from a young supernova remnant (SNR) with the Large Area Telescope aboard the Fermi Gamma-ray Space Telescope. These observations reveal a source with no discernible spatial extension detected at a significance level of $12.2 \sigma$ above $500 \mathrm{MeV}$ at a location that is consistent with the position of the remnant of the supernova explosion that occurred around 1680 in the Cassiopeia constellation-Cassiopeia A (Cas A). The gamma-ray flux and spectral shape of the source are consistent with a scenario in which the gamma-ray emission originates from relativistic particles accelerated in the shell of this remnant. The total content of cosmic rays (electrons and protons) accelerated in Cas A can be estimated as $W_{\mathrm{CR}} \simeq$ $(1-4) \times 10^{49}$ erg thanks to the well-known density in the remnant assuming that the observed gamma ray originates in the SNR shell(s). The magnetic field in the radio-emitting plasma can be robustly constrained as $B \geqslant 0.1 \mathrm{mG}$, providing new evidence of the magnetic field amplification at the forward shock and the strong field in the shocked ejecta.
\end{abstract}

Key words: acceleration of particles - ISM: individual objects (Cassiopeia A) - radiation mechanisms: non-thermal

Online-only material: color figures

\section{INTRODUCTION}

Supernova remnants (SNRs) have long been considered the primary candidates for the origin of Galactic cosmic rays (CRs). Specifically, diffusive shock acceleration (Bell 1978; Blandford \& Ostriker 1978; Jones \& Ellison 1991; Malkov \& O’C Drury $2001)$ is widely accepted as the mechanism by which charged particles can be accelerated to very high energies at collisionless shocks driven by supernova explosions. To maintain the energy density of the Galactic CRs, the kinetic energy released in supernova explosions has to be efficiently transferred to CRs with a conversion efficiency of $\sim 10 \%$ (Ginzburg \& Syrovatskii 1964). However, it has not yet been confirmed whether strong shock waves in SNRs are indeed capable of transferring this amount of energy into the acceleration of CR ions, and in doing so generating a CR energy density comparable to the energy density contained in the expansion ram pressure of the supernova.

Shock energy can be converted to another form of energy: turbulent magnetic fields. Recent theoretical work indicates that the magnetic field can be largely amplified by streaming of CRs themselves, as an integral part of efficient $\mathrm{CR}$ acceleration at collisionless shocks (Bell \& Lucek 2001; Bell 2004). Observations of synchrotron X-ray emission in young SNRs have shown that the magnetic field at supernova shocks can be am-

\footnotetext{
58 Royal Swedish Academy of Sciences Research Fellow, funded by a grant from the K. A. Wallenberg Foundation.
}

plified way beyond the factor of $\sim 4$ that is expected for standard compression of interstellar magnetic fields in the absence of CR modification of the shock's hydrodynamic structure (Uchiyama et al. 2007; Völk et al. 2005; Bamba et al. 2005; Vink \& Laming 2003). The amplified magnetic field would allow acceleration of protons up to the knee in the CR spectrum at $\sim 10^{15} \mathrm{eV}$, the presumed endpoint of the Galactic component (Bell \& Lucek 2001).

Cassiopeia A (Cas A) is the remnant of one of a handful of historical supernovae; the explosion around A.D. 1680 that gave rise to Cas A has probably been the last Galactic supernova witnessed by humans (Ashworth 1980). It is the brightest radio source in our Galaxy (Baars et al. 1977) and its overall brightness across the electromagnetic spectrum makes it a unique laboratory for studying high-energy phenomena in SNRs. Cas A has an angular size of 2'.5 in radius corresponding to a physical size of $2.34 \mathrm{pc}$ at a distance of $3.4_{-0.1}^{+0.3} \mathrm{kpc}$ (Reed et al. 1995). Cas A was the first SNR detected in TeV gamma rays, first by HEGRA (Aharonian et al. 2001) and later confirmed by MAGIC (Albert et al. 2007) and VERITAS (Humensky 2008), firmly establishing the existence of multi$\mathrm{TeV}$ particles. These higher-energy gamma rays are generally attributed to particles accelerated in the shock waves of the $\mathrm{SNR}$, even though the TeV gamma-ray source is not resolved. The emission mechanism(s) responsible for the TeV gammaray emission remained unsettled. EGRET reported only upper limits on the gamma-ray emission in the $\mathrm{GeV}$ band (Esposito et al. 1996). 
The advent of the Large Area Telescope (LAT) on board the Fermi Gamma-ray Space Telescope for the first time makes it possible to detect $\mathrm{GeV}$ gamma rays from the shell of Cas A. The relativistic bremsstrahlung of accelerated electrons has been predicted to lie above the sensitivity of the LAT (Atoyan et al. 2000). The prediction of the bremsstrahlung flux is controlled by the strength of the magnetic field and therefore the gamma-ray flux provides a measure of the amplified magnetic field in the supernova shell (Cowsik \& Sarkar 1980). InverseCompton (IC) scattering is another important mechanism of gamma-ray production by high-energy electrons; seed photons that can be upscattered to gamma rays include the interstellar radiation field, the cosmic microwave background (CMB), and the far-infrared (FIR) radiation by Cas A itself. In addition to these leptonic emissions, the decay of $\pi^{0}$ mesons produced in inelastic collisions between high-energy protons (and nuclei) and background gas may contribute to the $\mathrm{GeV}$ gamma-ray flux. The importance of the $\mathrm{GeV}$ observations of young SNRs is emphasized by the fact that the energy density of the accelerated particles is measurable thanks to the well-constrained gas and radiation density in Cas $\mathrm{A}$ in addition to that of the magnetic field. In this Letter, we report the discovery of GeV gammaray emission coincident with Cas A based on the first year observations with the Fermi LAT.

\section{OBSERVATIONS}

The LAT on board Fermi is a pair-conversion gamma-ray detector operating between $20 \mathrm{MeV}$ and $300 \mathrm{GeV}$. The LAT has a wide field of view of $\sim 2.4 \mathrm{sr}$ at $1 \mathrm{GeV}$, and observes the entire sky every two orbits ( $\sim 3 \mathrm{hr}$ for Fermi's orbit at an altitude of $\sim 565 \mathrm{~km}$ ). The full details of the instruments are given in Atwood et al. (2009).

A total exposure of $3 \times 10^{10} \mathrm{~cm}^{2} \mathrm{~s}$ (at $1 \mathrm{GeV}$ ) has been obtained for Cas A during the period between 2008 August 4 and 2009 September 4, corresponding to 394 days of observations. The data analysis was performed using the LAT Science Tools package with the P6_V3 post-launch instrument response function (Rando et al. 2009). The standard event selection for source analysis, resulting in the strongest background-rejection power (diffuse event class) was applied. In addition, photons coming from zenith angles larger than $105^{\circ}$ were rejected to reduce the background from gamma rays produced in the atmosphere of the Earth. The analysis was further restricted to the energy range above $200 \mathrm{MeV}$ where the uncertainties in the effective area become smaller than $10 \%$.

\section{ANALYSIS AND RESULTS}

The analysis of the gamma-ray event data was performed using gtlike, which is available as part of Fermi Science Tools. ${ }^{59}$ The tool gtlike employs a maximum likelihood technique to assess the statistical significance of sources and to estimate spectral parameters (Mattox et al. 1996). The background gammaray model includes background sources from the 11 month catalog of Fermi-LAT sources at fixed coordinates, the galactic diffuse emission (using gll_iem_v02.fit), and an isotropic component (using isotropic_iem_v02.txt). The maximum likelihood analysis is performed inside a region of interest (ROI) of $10^{\circ}$ radius centered on Cas $\mathrm{A}$. The independent tool sourcelike was employed to determine the source position and constrain the

\footnotetext{
59 Software and documentation of the Fermi Science Tools are distributed by the Fermi Science Support Center at http://fermi.gsfc.nasa.gov/ssc.
}

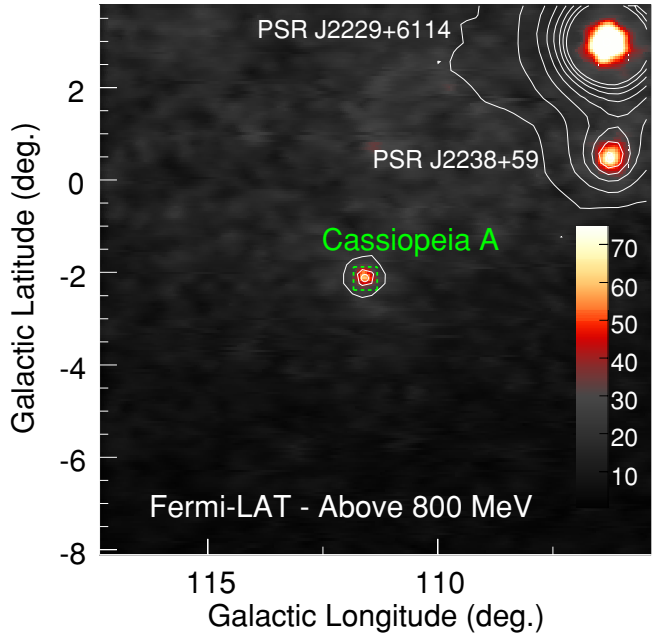

Figure 1. Smoothed gamma-ray count map of the region surrounding Cas A with side length $12^{\circ}$, binned in square pixels of side length 0.05 . Smoothing was done with a Gaussian of width 0.1 . Overlaid are test statistics contours (TS $=-2 \ln (-L)$ ) for the assumption of a point source located at the position of the pixel. Contours of 25, 100, 200, 300, 400, 500, 600, and 700 TS levels are shown. Only events with energies larger than $800 \mathrm{MeV}$ were used.

(A color version of this figure is available in the online journal.)

angular extent of the source. Sourcelike performs a maximum likelihood fit to simultaneously optimize position and extent of the source (given an assumption about the shape of the source) in independent energy bands. Sourcelike can also be used to assess the test-statistic (TS) value and to compute the spectra of both extended and point sources. The position determined with sourcelike is consistent with what was found by the automated LAT catalog tool. The spectral parameters between sourcelike and gtlike for this best-fit position are found to be consistent, yielding an independent check of the main results of the Letter.

The analysis clearly shows a source above the background coincident with the SNR Cas A (see Figure 1). The source is detected at a significance level of $12.2 \sigma$ (or a TS value of 148) above the background at a best-fit position of $\alpha_{2000}=$ $23^{\mathrm{h}} 23^{\mathrm{m}} 17.5, \delta_{2000}=58^{\mathrm{h}} 49^{\mathrm{m}} 43^{\mathrm{s}} .1$ with a statistical uncertainty on the location of 1.0 (68\% confidence level). The systematic error in the position due to alignment of the telescope system and inaccurate description of the point-spread function of the instrument is estimated to be 0.3 . The position is spatially coincident with Cas A and with the MAGIC and VERITASdetected TeV gamma-ray source (Albert et al. 2007; Humensky 2008) as shown in Figure 2. An upper limit on the size of the gamma-ray emission has been obtained in a maximum likelihood fit by investigating the decrease of the likelihood with increasing source size. Under the assumption of a Gaussian shape, this $68 \%$ upper limit amounts to 3.5 ( $1 \sigma$ radius). This value is larger than the radius of the SNR (2.5), which thus currently cannot be resolved by the Fermi LAT. A check for variability in this data set with one-month time bins shows no sign for any change in the gamma-ray flux with time, indicating a steady source of emission.

A spectral analysis of the gamma-ray emission has been performed in an energy range of $200 \mathrm{MeV}-50 \mathrm{GeV}$. The source is detected only above $500 \mathrm{MeV}$. The spectrum shown in Figure 3 reveals a relatively flat energy distribution and can be fitted by a power law with a spectral index of $\Gamma=2.0 \pm 0.1$ between $0.5 \mathrm{GeV}$ and $50 \mathrm{GeV}$. The systematic uncertainty in the spectral index determination from the uncertainty in the normalization 


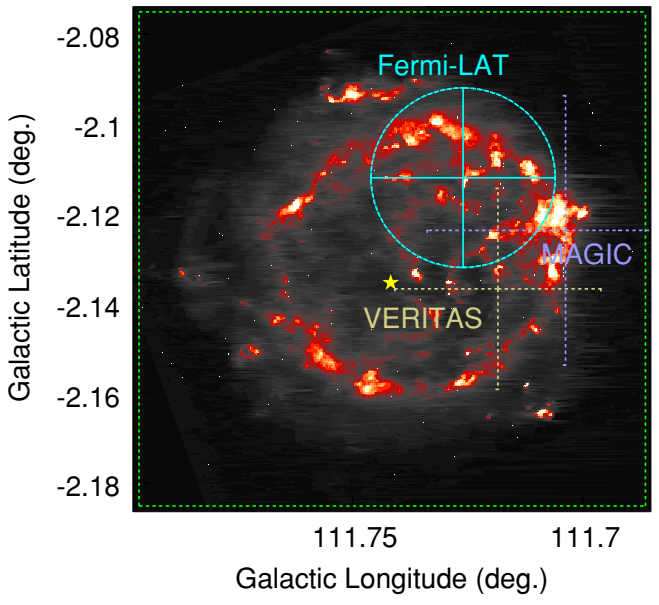

Figure 2. VLA $20 \mathrm{~cm}$ radio map of the Cas A supernova shell (Anderson \& Rudnick 1995). Shown is the region corresponding to the green square in Figure 1. The graph shows the $\mathrm{GeV}$ position with error bars (conservatively adding statistical and systematic errors in quadrature) as a cyan circle and the position of the CCO as a yellow star. Also shown in the plot are the best-fit positions for MAGIC (Albert et al. 2007) and VERITAS (Humensky 2008).

(A color version of this figure is available in the online journal.)

of the Galactic diffuse emission (conservatively estimated to be $10 \%$ ) amounts to $\sim 0.1$. The integral photon flux above $500 \mathrm{MeV}$ amounts to $(8.7 \pm 1.3) \times 10^{-9}$ photons $\mathrm{cm}^{-2} \mathrm{~s}^{-1}$. A likelihood-ratio test was performed to check the presence of a spectral cutoff using a spectral function of $d N / d E=$ $K E^{-\Gamma} \exp \left(-E / E_{\text {cut }}\right)$. The presence of the exponential cutoff is not statistically significant, given the resultant likelihood ratio of $-2 \ln \left(L_{\mathrm{PL}} / L_{\text {cutoff }}\right)=2.6$. We formally report the best-fit parameters of the cutoff power-law model to make a comparison with LAT pulsars: $\Gamma=1.7 \pm 0.2$ and $E_{\text {cut }}=16 \pm 9 \mathrm{GeV}$.

\section{DISCUSSION}

The detection of gamma-ray emission from the direction of Cas A raises the following questions: "Is there a compact source such as a pulsar dominating the emission"? and "What is the radiating particle population responsible for the emission?" Two emission scenarios seem plausible: emission from the central compact object (CCO) in Cas A or emission generated in the SNR. Since the GeV gamma-ray source coinciding with Cas A is a point source in the LAT, these two scenarios cannot be distinguished on positional grounds; therefore, circumstantial evidence has to be considered.

The point-like central X-ray source (Tananbaum 1999) is generally thought to be the left-over of the explosion of the massive progenitor star (Pavlov et al. 2000) and known as a CCO given that it is radio quiet, un-pulsed in X-rays, and has an X-ray spectrum described by blackbody with characteristic temperatures of about $0.4 \mathrm{keV}$ without indication of a nonthermal component. A recent Chandra observation resulted in a $3 \sigma$ limit on the pulsed fraction of $16 \%$ for periods larger than $0.68 \mathrm{~s}$ (Pavlov \& Luna 2009). The fact that no pulsation has been detected at any waveband for the $\mathrm{CCO}$ does not rule out that the neutron star is emitting gamma rays. The Fermi LAT has detected several neutron stars as gamma-rayonly pulsars, pulsars not previously known from observations in other wavebands (Abdo et al. 2008, 2009a).

We searched for gamma-ray pulsations from the source coincident with Cas A using the full data set. We applied the standard time-differencing technique (Atwood et al. 2006),

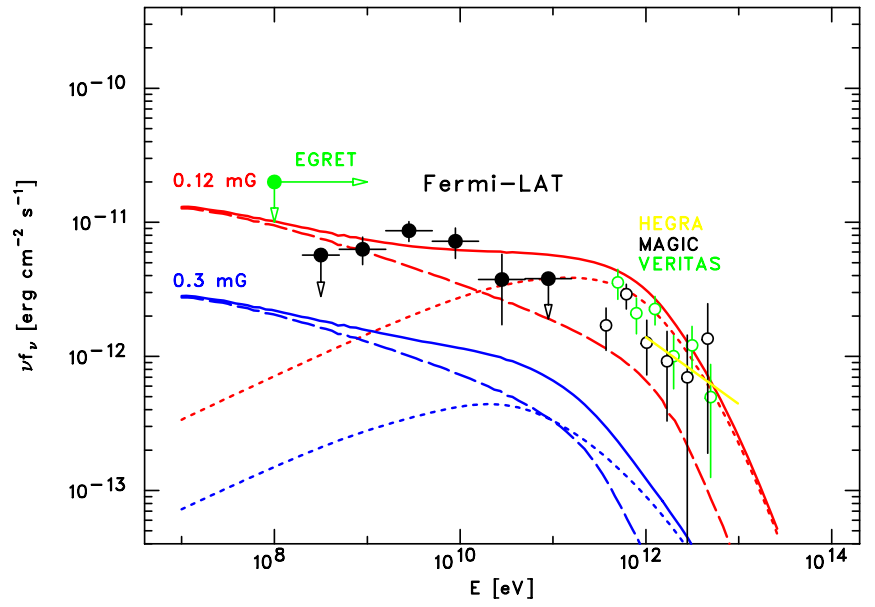

Figure 3. Energy spectrum of Cas A in a leptonic emission model. Shown is the Fermi-LAT detected emission (filled circles) in comparison to the energy spectra detected by MAGIC (black open circles; Albert et al. 2007) and VERITAS (green open circles; Humensky 2008). The red curves show a leptonic model calculated for $B=0.12 \mathrm{mG}$ while the blue curves show one for $B=0.3 \mathrm{mG}$. Dashed lines show the bremsstrahlung components with $n_{\mathrm{eff}} \simeq 26 \mathrm{~cm}^{-3}$, and dotted lines show the IC component.

(A color version of this figure is available in the online journal.)

using a maximum frequency of $64 \mathrm{~Hz}$, and a long timedifference window of $\sim 12$ days. We found no evidence for pulsations which makes the neutron star scenario less likely. A comparison of the blind search pulsars discovered so far (Abdo et al. 2009a) and the known radio pulsars detected by the LAT, suggests that the blind search is approximately $2-3$ times less sensitive than a standard pulsation search using the known timing solution. This results in a $5 \sigma$ limit on the pulsed flux of $\sim 2 \times 10^{-7}$ photons $\mathrm{cm}^{-2} \mathrm{~s}^{-1}$ (Abdo et al. 2009b). In addition, there is no $\mathrm{GeV}$ gamma-ray source in the first Fermi-LAT source catalog that is associated with a known CCO.

Furthermore, the spectrum does not support a pulsar hypothesis. The energy spectra of pulsars are usually flat at energies below $1 \mathrm{GeV}$ and show exponential cutoffs in the energy range between $1 \mathrm{GeV}$ and $8 \mathrm{GeV}$ (Abdo et al. 2009b). These characteristics do not mirror the LAT spectrum which is best described by a power-law shape with an index of $\Gamma=2.0 \pm 0.1$ up to $50 \mathrm{GeV}$ with no significant sign of a high-energy cutoff. A formal fit with an exponential cutoff model yields $E_{\text {cut }}=16 \pm 9 \mathrm{GeV}$. This is rather high for a gamma-ray pulsar; no LAT pulsars show $E_{\text {cut }}>8 \mathrm{GeV}$ so far (Abdo et al. 2009a).

The scenario in which the $\mathrm{GeV}$ gamma rays are emitted in the shell of the SNR is therefore favored. The gamma-ray emission could be produced by electrons accelerated at the forward shock through relativistic bremsstrahlung or IC. Alternatively, the $\mathrm{GeV}$ gamma-ray emission could be predominantly produced by accelerated hadrons through interaction with the background gas and subsequent $\pi^{0}$ decay. Recent studies showed electron acceleration to multi- $\mathrm{TeV}$ energies is likely to take place also at the reverse shock in the supernova ejecta (Uchiyama \& Aharonian 2008; Helder \& Vink 2008).

First, we consider the shocked circumstellar region between the contact discontinuity and the forward shock (see Gotthelf et al. 2001), assumed to have a constant magnetic field of $B$ (a free parameter), and a shocked circumstellar medium with a constant density of $n_{\mathrm{H}}=10 \mathrm{~cm}^{-3}$ (Laming \& Hwang 2003). Electrons are accelerated to multi-TeV energies at the forward shock as traced by synchrotron X-ray outer filaments (Hughes et al. 2000). Here we adopt an electron acceleration 
spectrum $Q_{\mathrm{e}}(E) \propto E^{-2.34} \exp \left(-E / E_{\mathrm{m}}\right)$ to match the radioIR spectral index of $\alpha=0.67$ (Rho et al. 2003), since both the GeV gamma-ray emission and the radio synchrotron emission sample similar electron energies. About half of the total radio flux is attributable to this region (the so-called plateau), while another half is to the reverse shock region (the so-called bright ring; see below). Given the radio flux and the effective density $\left(n_{\text {eff }}=\Sigma n_{i} Z_{i}\left(Z_{i}+1\right) \simeq 26 \mathrm{~cm}^{-3}\right)$, the flux of bremsstrahlung is controlled by $B$. In Figure 3, we show a leptonic model with $B=0.12 \mathrm{mG}$ (red curves), which can broadly explain the observed GeV flux. The maximum energy is set to be $E_{\mathrm{m}}=40 \mathrm{TeV}$ (Vink \& Laming 2003). Shown are contributions from bremsstrahlung (dashed) and from IC scattering (dotted) produced by accelerated electrons that suffer synchrotron cooling at high energies. The bremsstrahlung spectrum consisting of electron-ion and electron-electron components is computed as in Baring et al. (1999). The radiation field for the IC component is dominated by FIR emission from the Cas A ejecta, characterized by a temperature of $100 \mathrm{~K}$ and an energy density of $\sim 2 \mathrm{eV} \mathrm{cm}^{-3}$ (Mezger et al. 1986), a factor of 8 larger than the energy density in the CMB. The IC/FIR emission exceeds IC/ $\mathrm{CMB}$ by a factor of 2.7 at a gamma-ray energy of $10 \mathrm{GeV}$. The value of $B=0.12 \mathrm{mG}$ is consistent with $B=0.08-0.16 \mathrm{mG}$ at the forward shock estimated by Vink \& Laming (2003) based on the width of a synchrotron X-ray filament. Note, however, that a somewhat higher value of $B \simeq 0.3 \mathrm{mG}$ was obtained by Parizot et al. (2006) using the same filament width but including projection effects. The total amount of electrons in this case is $W_{e}(>10 \mathrm{MeV}) \simeq 1 \times 10^{49} \mathrm{erg}$. Also shown in Figure 3 is the case of $B=0.3 \mathrm{mG}$ (blue curves), which predicts a lower gamma-ray flux than the observed one.

In a scenario in which the gamma rays are generated by $\pi^{0}$ decay of accelerated hadrons, the gamma-ray spectrum can be well matched with either the proton acceleration spectrum $Q_{\mathrm{p}}(p) \propto p^{-2.3}$ (a red curve in Figure 4), or a harder proton spectrum of $Q_{\mathrm{p}}(p) \propto p^{-2.1}$ with an exponential cutoff at $10 \mathrm{TeV}$ that is arbitrarily introduced (blue curve). Here $p$ denotes momentum of accelerated protons. The gamma-ray spectrum is calculated following Kamae et al. (2006) with a scaling factor of 1.85 for helium and heavy nuclei (Mori 2009). The total proton content amounts to $W_{p}\left(>10 \mathrm{MeV} \mathrm{c}^{-1}\right) \simeq 3.8 \times 10^{49} \mathrm{erg}$ in the case of the softer spectrum and to $W_{p}\left(>10 \mathrm{MeV} c^{-1}\right) \simeq$ $3.2 \times 10^{49} \mathrm{erg}$ in the case of the harder proton spectrum with the cutoff. In both cases, the energy content corresponds to less than $2 \%$ of the estimated explosion kinetic energy of $E_{\text {sn }}=2 \times 10^{51} \mathrm{erg}$. Therefore, the CR pressure would not be large enough to change the hydrodynamics of Cas A. This is consistent with Chandra X-ray measurements of the remnant's spatial structure (e.g., Gotthelf et al. 2001); the ratio of the radii of forward and reverse shocks can be reproduced by hydrodynamical models that do not include CR acceleration (Laming \& Hwang 2003).

Comparing the leptonic and hadronic models, it seems clear that the hadronic scenario can better fit the data due to the turnover at low energies that is not well reproduced in the leptonic scenario. Given the uncertainties in the diffuse model that have stronger effects at the low-energy end, we refrain, however, from strong claims about the radiating particle population at this point. Changing the diffuse model normalization by $\pm 10 \%$ (a conservative assumption on the uncertainty) largely affects the energy points at and below $1 \mathrm{GeV}$. The resulting effect on the flux point at $1 \mathrm{GeV}$ is a $25 \%$ upward and $65 \%$ downward shift.

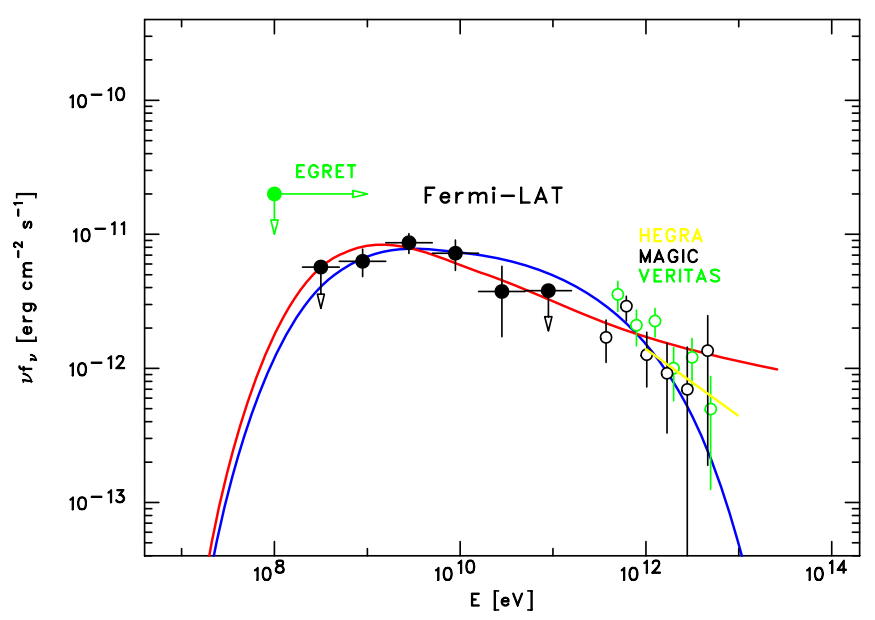

Figure 4. Same as Figure 3 but in a hadronic emission model. Shown are $\pi^{0}$ decay spectra for two possible proton spectra. The blue line shows a harder proton index of 2.1 with a cutoff energy of $10 \mathrm{TeV}\left(W_{p}=3.2 \times 10^{49} \mathrm{erg}\right.$ for $\left.n_{\mathrm{H}}=10 \mathrm{~cm}^{-3}\right)$. The red line shows a softer proton index of 2.3 without a cutoff. (A color version of this figure is available in the online journal.)

A more detailed investigation of the lower energy end of the LAT spectrum will be possible with future LAT data.

The shocked ejecta gas that emits strong radio and infrared synchrotron light (known as the bright ring) is another potential gamma-ray emitting region. Using $M_{\text {ejecta }}=2 M_{\odot}$ (Willingale et al. 2002; Laming \& Hwang 2003) composed of only oxygen, we obtain $n_{\text {eff }}=n_{\mathrm{O}} Z_{\mathrm{O}}\left(Z_{\mathrm{O}}+1\right) \simeq 32 \mathrm{~cm}^{-3}$. This happens to be similar to the value of $n_{\text {eff }}$ in the forward shock region. Also, the baryon density of $n_{\mathrm{O}} A_{\mathrm{O}} \simeq 7 \mathrm{~cm}^{-3}$ is close to that in the forward shock region. Therefore, the $\pi^{0}$ decay predictions are essentially the same as those described above, though the energy budget is tighter in the reverse shock. The total thermal energy stored in the shocked ejecta would be only $\sim 1 \times 10^{50} \mathrm{erg}$. On the other hand, a combination of $n_{\text {eff }} \simeq 32 \mathrm{~cm}^{-3}$ and the large magnetic field in the shocked ejecta $(B \gtrsim 0.5 \mathrm{mG})$ inferred by X-ray variability (Uchiyama \& Aharonian 2008) makes it difficult to attribute the gamma-ray emission to the relativistic bremsstrahlung in the shocked ejecta.

Regardless of the origin(s) of the observed gamma rays, the total content of CRs accelerated in Cas A can be obtained as $W_{\mathrm{CR}}=W_{e}+W_{p} \simeq(1-4) \times 10^{49} \mathrm{erg}$, and the magnetic field amplified at the shock and the field in the shocked ejecta can be constrained as $B \geqslant 0.12 \mathrm{mG}$. Even though Cas $\mathrm{A}$ is considered to have entered the Sedov phase, the total amount of CRs accelerated in the remnant constitutes only a minor fraction $(\leqslant 2 \%)$ of the total kinetic energy of the supernova. The bremsstrahlung spectrum and the $\pi^{0}$ decay spectrum have rather different predictions below $1 \mathrm{GeV}$. The hard spectrum below $1 \mathrm{GeV}$ would favor the $\pi^{0}$ decay origin, though the current LAT data quality does not rule out the bremsstrahlung model.

The Fermi LAT Collaboration acknowledges support from a number of agencies and institutes for both development and the operation of the LAT as well as scientific data analysis. These include NASA and DOE in the United States, CEA/Irfu and IN2P3/CNRS in France, ASI and INFN in Italy, MEXT, KEK, and JAXA in Japan, and the K. A. Wallenberg Foundation, the Swedish Research Council, and the National Space Board in Sweden. Additional support from INAF in Italy and CNES in 
France for science analysis during the operations phase is also gratefully acknowledged.

\section{REFERENCES}

Abdo, A. A., et al. (The Fermi LAT Collaboration) 2008, Science, 322, 1218 Abdo, A. A., et al. (The Fermi LAT Collaboration) 2009a, Science, 325, 840 Abdo, A. A., et al. (The Fermi LAT Collaboration) 2009b, ApJ, submitted (arXiv:0910.1608)

Aharonian, F., et al. (The HEGRA Collaboration) 2001, A\&A, 370, 112

Albert, J., et al. (The MAGIC Collaboration) 2007, A\&A, 474, 937

Anderson, M. C., \& Rudnick, L. 1995, ApJ, 441, 307

Ashworth, W. B. 1980, J. Hist. Astron., 11, 1

Atoyan, A. M., Aharonian, F. A., Tuffs, R. J., \& Völk, H. J. 2000, A\&A, 355, 211

Atwood, W. B., et al. 2006, ApJ, 652, L49

Atwood, W. B., et al. (The Fermi LAT Collaboration) 2009, ApJ, 697, 1071

Baars, J. W. M., Genzel, R., Pauliny-Toth, I. I. K., \& Witzel, A. 1977, A\&A, 61, 99

Bamba, A., Yamazaki, R., Yoshida, T., Terasawa, T., \& Koyama, K. 2005, ApJ, 621,793

Baring, M. G., Ellison, D. C., Reynolds, S. P., Grenier, I. A., \& Goret, P. 1999, ApJ, 513, 311

Bell, A. R. 1978, MNRAS, 182, 147

Bell, A. R. 2004, MNRAS, 353, 550

Bell, A. R., \& Lucek, S. G. 2001, MNRAS, 321, 433

Blandford, R. D., \& Ostriker, J. P. 1978, ApJ, 221, L29

Cowsik, R., \& Sarkar, S. 1980, MNRAS, 191, 855

Esposito, J. A., Hunter, S. D., Kanbach, G., \& Sreekumar, P. 1996, ApJ, 461, 820

Ginzburg, V. L., \& Syrovatskii, S. I. 1964, The Origin of Cosmic Rays (New York: Macmillan)
Gotthelf, E. V., Koralesky, B., Rudnick, L., Jones, T. W., Hwang, U., \& Petre, R. 2001, ApJ, 552, L39

Helder, E. A., \& Vink, J. 2008, ApJ, 686, 1094

Hughes, J. P., Rakowski, C. E., Burrows, D. N., \& Slane, P. O. 2000, ApJ, 528, L109

Humensky, T. B. 2008, in AIP Conf. Ser. 1085, 4th Heidelberg International Symp. on High Energy Gamma-Ray Astronomy, ed. F. A. Aharonian, W. Hofmann, \& F. Rieger (Melville, NY: AIP), 357

Jones, F. C., \& Ellison, D. C. 1991, Space Sci. Rev., 58, 259

Kamae, T., Karlsson, N., Mizuno, T., Abe, T., \& Koi, T. 2006, ApJ, 647, 692

Laming, J. M., \& Hwang, U. 2003, ApJ, 597, 347

Malkov, M. A., \& O’C Drury, L. 2001, Rep. Prog. Phys., 64, 429

Mattox, J. R., et al. 1996, ApJ, 461, 396

Mezger, P. G., Tuffs, R. J., Chini, R., Kreysa, E., \& Gemuend, H.-P. 1986, A\&A, 167,145

Mori, M. 2009, Astropart. Phys., 31, 341

Parizot, E., Marcowith, A., Ballet, J., \& Gallant, Y. A. 2006, A\&A, 453, 387

Pavlov, G. G., \& Luna, G. J. M. 2009, ApJ, 703, 910

Pavlov, G. G., Zavlin, V. E., Aschenbach, B., Trümper, J., \& Sanwal, D. 2000, ApJ, 531, L53

Rando, R., et al. (The Fermi LAT Collaboration) 2009, in Proc. 31st International Cosmic Ray Conf., in press (arXiv:0907.0626)

Reed, J. E., Hester, J. J., Fabian, A. C., \& Winkler, P. F. 1995, ApJ, 440, 706

Rho, J., Reynolds, S. P., Reach, W. T., Jarrett, T. H., Allen, G. E., \& Wilson, J. C. 2003, ApJ, 592, 299

Tananbaum, H. 1999, IAU Circ., 7246, 1

Uchiyama, Y., \& Aharonian, F. A. 2008, ApJ, 677, L105

Uchiyama, Y., Aharonian, F. A., Tanaka, T., Takahashi, T., \& Maeda, Y. 2007, Nature, 449, 576

Vink, J., \& Laming, J. M. 2003, ApJ, 584, 758

Völk, H. J., Berezhko, E. G., \& Ksenofontov, L. T. 2005, A\&A, 433, 229

Willingale, R., et al. 2002, A\&A, 398, 1021 